\title{
A Risk Analysis Method for Selecting Logistic Partners to Compose Virtual Organizations
}

\author{
Omir Correia Alves Junior ${ }^{1}$, Ricardo J. Rabelo ${ }^{2}$, \\ Rafael Giordano Vieira ${ }^{1}$, and Adriano Fiorese ${ }^{1}$ \\ ${ }^{1}$ Dept. of Computer Science, State University of Santa Catarina, Brazil \\ ${ }^{2}$ Dept. of Automation and Systems Eng., Federal University of Santa Catarina \\ \{omalves, rafaelgiordano12, adriano.fiorese\} @gmail.com, \\ rabelo@das.ufsc.br
}

\begin{abstract}
Virtual Organizations (VO) have complex interactions between their members, both industrial and logistics. Once industrial partners are selected, the VO should be complemented with the logistics partners that will deliver the produced goods between those partners. This paper presents a supporting decision method for selecting the most suitable logistics partners that consider not only capabilities, historical performance and availability, but also risks. In general, a risk is characterized by the potential of each logistic partner to do not fulfill VO's business requirements and that can jeopardize its accomplishment. The method firstly evaluate and measure risks per partner, and collectively afterwards. Its formalization and examples are provided along the paper. Discussions are presented in the end.
\end{abstract}

Keywords: virtual organization, logistics partners' selection, KPI, risk analysis.

\section{Introduction}

Companies have increasingly been immersed in more dynamic and adaptive value chain networks so favoring the expansion of logistic partners. New markets and new products have been increasingly created all over the world and proper logistic partners should be hired in order to cope with this need. The cleverer the hiring activity is done, the greater visibility, improved customer service, better planning and cost savings can be supported [1]. This cleverness can also be crucial for companies as a support for reindustrialization and for facing current economic challenges. However, when companies get involved in more volatile strategic networks, the difficulty of selecting the most appropriate logistic partners is much higher.

This work focuses on the Virtual Organization (VO) type of strategic network. A $\mathrm{VO}$ is a temporary and dynamic alliance of autonomous, heterogeneous and usually geographically dispersed companies (often SMEs) created to attend to certain demands [2], sharing costs, benefits and risks, acting as they were one single enterprise [3]. A VO dismisses itself after ending all its legal obligations. Therefore, part of that difficulty in terms of logistics is due to the VO intrinsic nature, that industrial partners are only known after an analysis of the demand's requirements. 
Once industrial partners are selected and hence the VO is formed, it should then be complemented with logistics partners (LP). By LP it is considered in this work the logistics providers (of types $2 L P$ and $3 P L$ ) which act at the 'arc' part of the network (outbound) and that are totally responsible for delivering the produced goods between VO's industrial partners.

In a previous work authors conceived a model to select LPs [4]. It applied fifteen key performance indicators (KPI) over the set of possible LPs to further select the most adequate ones for each logistic leg considering the VO's industrial partners and the whole logistics path. This selection was based on the LPs' historical performance in past VOs, on their technical competence and temporal availability, and on the demand's requirements.

The problem is that, even considering such elements, there is a risk of failure. Faults in some LP can affect other partners and lead the given VO to fail in its goals [6]. Therefore, it is very important to measure them for further decision-making.

In this sense, this paper presents a complementary work to also take risks into account. Considering a sort of potential sources of risks, the VO risk of each possible composition of LPs is calculated so that the responsible stakeholders can select the most qualified and less risky list of LPs for a given VO before it goes for operation. Besides that, they can further act towards mitigating risks if it was the case.

This work presents results of an ongoing, applied and qualitative research which basically look for answering how LPs for a given VO can be more properly measured when considering risks. It is represented by a novel method for risk analysis in the formation of VOs called MRALP (Multi criteria Risk Analysis method applied to Logistic Partner). ETA and FTA risk analysis methods [7, 8] are the essential theoretical basis for the proposed method.

In this work the current focus is only on LPs, not covering yet the VO as a whole, i.e. the industrial partners as well. Besides that, it assumes that LPs are be members of long-term alliances (like VBE [3]) so sharing some minimum and common collaboration, working, quality and performance principles.

This paper is organized as follow: Section 1 has introduced the general problem and paper's goal. Section 2 addresses the problem of selection of LPs for VO and contextualizes it within the risk analysis area. Section 3 presents the proposed method. Section 4 shows an example of the method. Finally, Section 5 provides preliminary conclusions about this research.

\section{Risk Analysis in Virtual Organizations}

There are many definitions of what risk means in the literature. In resume, risk can be defined as the probability of an event to can occur and that causes a negative or positive impact on the organization's goals when it takes place. A risk can be viewed as a composition of three basic elements: the general environment within it can happen; its probability of occurrence; and the scope of its impact in the case of its occurrence [7]. In the context of this research, a risk is characterized by the potential of each LP does not cope with the given demand / collaboration opportunity's (CO) requirements and hence can jeopardize the VO accomplishment. Thus, it is necessary to comprehensively identify and quantify the VO risks associated to the selected LPs. 
In the research review a number of risk analysis methods has been identified as potentially suitable for VOs, namely FMEA (Failure mode and effects analysis), FTA (Fault Tree Analysis), ETA (Event Tree Analysis), Bayesian Networks, CNEA (Causal Network Event Analysis) and Ishikawa Diagram [7,8,9,10,11,12]. Some requirements can be pointed out for the tackled problem [5]: events can be treated as independent from each other; the deterministic relation between events can be known; events analysis can be both qualitative and quantitative; a risk can be globally quantified after a succession of events. Regarding these requirements, ETA and FTA techniques were selected to be used and combined in the proposed method.

In a review of the state-of-the-art some works related to risk analysis for VOs failures have been identified. In [13,14] thirteen KPIs were identified as general risk sources in VOs, further identifying the importance of each one. In [15] two sources of risks were specified (external and internal) and risk occurrence likelihood in the life span of a VO was calculated based on them. In [6] the problem of risk mitigation in VO was discussed and four processes were identified to improve the level of VO performance reliability. In spite of the value of their results and that some insights have been taken from them to this work, none of them has neither somehow formalized how the proposed KPIs should be used nor provided means to quantity VO partners' risks, both individually and collectively. Actually, it was not found out any work in the literature that has tackled logistics issues and measured VO risks.

Considering the classical main phases of a VO life cycle (creation, operation, evolution and dissolution phases) [3], risk analysis should ideally be done along the entire process. The scope of this research is however focused on the creation phase, i.e. to provide means to managers to evaluate the VO before its operation.

Within the creation phase, and respecting the reference framework for the VO creation [3], it seems more natural that risk analysis be carried out in the Partners' Search and Selection step, i.e. after the VO topology are defined and before selected partners starting eventual negotiations. In [16] authors expanded this step to introduce a process of selecting the proper performance indicators that should be applied over candidate industrial partners' (IP) for a VO regarding the CO. In [4] this same framework was complemented and expanded again to comprise the selection of logistics partners (Figure 1). The work presented in this paper introduces an additional sub-step within this part in order to embrace risk analysis (circle in Figure 1).

When related to VOs, risks can be identified and measured considering the equivalent factors that determine the risk probability in traditional organizations [6]. As such, they should consider the organization's goals so that the most important ones can be determined for more proper management. Identifying risk sources is the first and crucial step in risk management [7].

Although risk analysis is performed in the Creation phase, sources of risks should be identified and risks measured having in mind the whole VO life cycle [15]. There are four main sources of risks regarding VOs: trust, communication, collaboration and commitment $[13,14]$. In this work they are modeled as KPIs and their values are calculated and provided by the previous method phase, developed in [4].

- Trust: Logistic partners who are going to compose a VO do not necessarily have prior knowledge about each other before starting collaborating. Thus trust is crucial to bear in mind, which in turn involves commitment in doing the planned 
tasks. When trust among partners is not enough established there is a hesitation to share risks and so the VO can be jeopardized.

- Communication: Communication among VO's LPs is a key factor for its proper operation. They should provide correct information about parts, products and services, collaborating in solving conflicts, sharing practices, etc. However, this can be complicated by the fact that LPs are heterogeneous, independent, geographically dispersed and usually have distinct working cultures. The insufficient communication can put a VO on risk.

- Collaboration: Collaboration is characterized when the sharing of risks, costs and benefits of doing business are agreed and fairly distributed among partners. However, when a collaboration agreement is not clearly defined, i.e., when there is no clear definition of its main objectives, the risk of a VO increases.

- Commitment: Commitment is related to the attitude of VO partners with each other, i.e. it considers the contributions and agreements made by and among them for a business. This is important as partners use to have complementary skills and so it is important they feed the whole environment with the right and timely information. The VO risk gets higher when partners fail in that attitude.

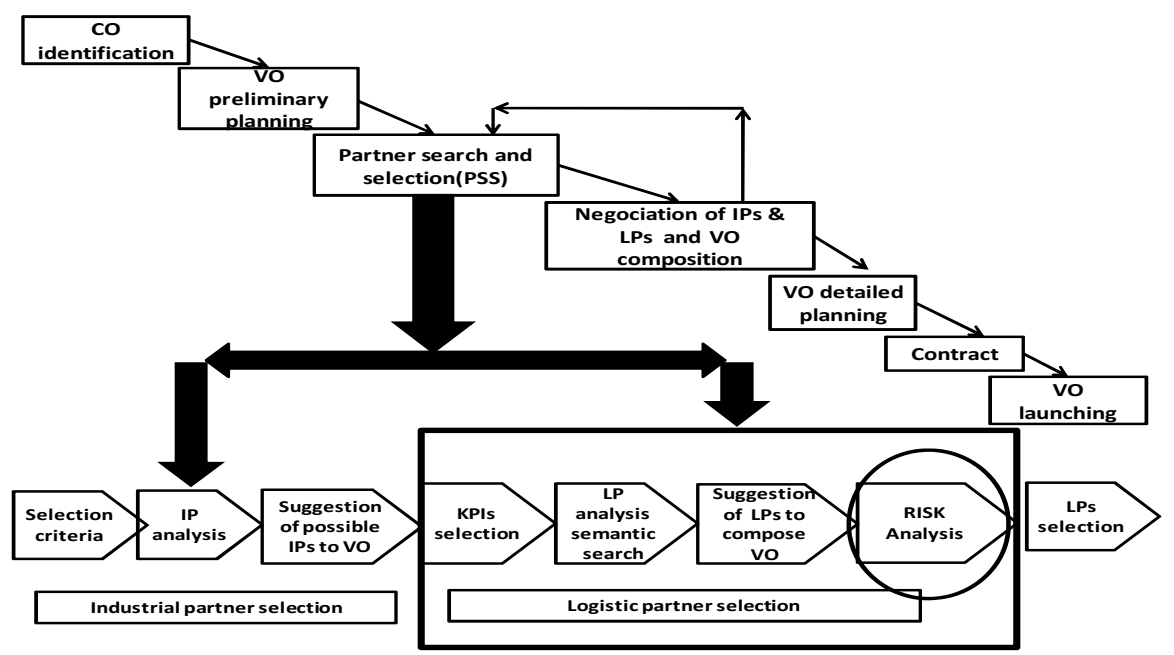

Fig. 1. Extended framework for the VO creation [4]

\section{The Proposed Risk Analysis Method}

In general, the most important requirement for any method related to VOs is transparency, which is a basis for trust building among partners. In this direction, being a selection (or suggestion) process, this work helps in leaving clear the criteria for that as well as in providing a more systematic process, i.e. a formal method.

The devised model for risk analysis starts having as input a pre-selected and ranked list of most suitable LPs for each itinerary within the given VO. As mentioned in the introduction, this pre-selection is performed by the previous phase of the method [4] 
involving a set of elements. In this work, considering VO reference foundations [3], the so-called VO Manager is seen as the main decision-maker.

The proposed method is generally presented in Figure 2. It splits the problem into two stages. It starts measuring the risks individually, for each of pre-selected LP, and after and based on that, collectively, for the entire LP team for the given VO. In this context, VO manager has a fundamental role, which is to define (with some degree of subjectivity) the minimal acceptable level (MAL) of risk for a LP to be member of a VO regarding CO's requirements and the strategic vision upon the VO. There is also a risk specialist, who is in charge of auditing the LPs' historical KPI values at the VBE's data repository.

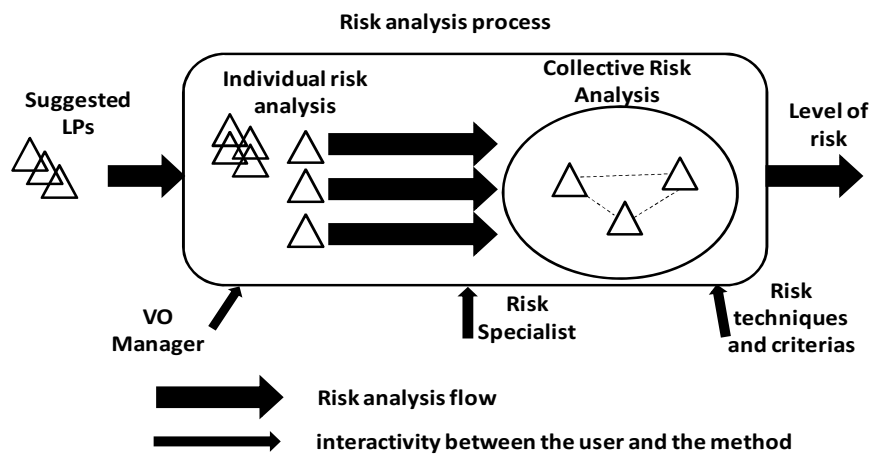

Fig. 2. Risk Analysis model

The MRALP method itself is illustrated in Figure 3. Inspired in [17], it divides the problem into two phases: the first phase does the individual risk analysis and applies ETA method for that. The second phase does the risk analysis taking the group of LPs as a whole into account, applying FTA method.

\subsection{Individual Risk Analysis}

In the first phase of MRALP an individual risk analysis for pre-selected LPs is performed. ETA is particularly suitable for risk analysis of systems where there are interactions between several types of probabilistic events, whether dependent or independent [8]. It uses a visual representation based on a logical binary tree structure, known as Event Tree (ET), as shown in Figure 3 (Stage 1).

An ET is a probability tree which provides two possible conditions: success and failure. It also has three basic components: initiating event; intermediary events; and outcomes. The initiating event begins the ET creation process. In this work, the initiating event corresponds to one pre-selected LP, and the assigned probability is always 1 (or 100\%) in the beginning [8]. Next step consists in specifying the (four) intermediary events, which are represented by the (four) KPIs: trust, communication, collaboration and commitment. These events are used to quantify the effectiveness of a particular LP, i.e. if it is able or not to compose a VO. 
These KPIs are used to generate an ET by assigning success and failure probabilities to each of them, as shown in Figure 3 (Stage 1). Inspired in [18], the criterion to assign the KPI success probability to each LP takes the historical values analysis of the KPI that were assigned to it in past VOs. The proposed method gets these KPI values and checks if they are greater than MPS (Minimum Probability of Success). MPS is a value that is also set up by the VO manager and represents the minimum probability of a KPI value to be within the considered acceptable range. For instance, in Figure 4, the value of the MPS related to the (intermediate event 'KPI_1') trust would have been set up as 0.3 (he also gives MPS values to the other three KPIs). In this figure, the VO manager would be stating that he accepts a global risk of 0,4 (MAL) and that the minimum level (of fail) for trust is 0,3 and so 0.7 for success.

The setting up of MAL and MPS are a bit subjective, and basically tries to make a trade-off between acceptable risk and effective performance situation of existing LPs. In the same line, each VBE can adopt a particular way of calculating every KPI, usually grouping a set of performance indicators at the operational level. In this case, the following understanding would be used by the given VBE's partners when classifying their KPIs: [0.0; 0.2]: regrettable; $[0.2 ; 0.4]$ : bad; [0.4;0.6]: regular; $[0.6 ; 0.8]$ : good; $[0.8 ; 1.0]$ : superior.

Applying Equation 1 (see below), the KPI success probability is calculated for the current participation.

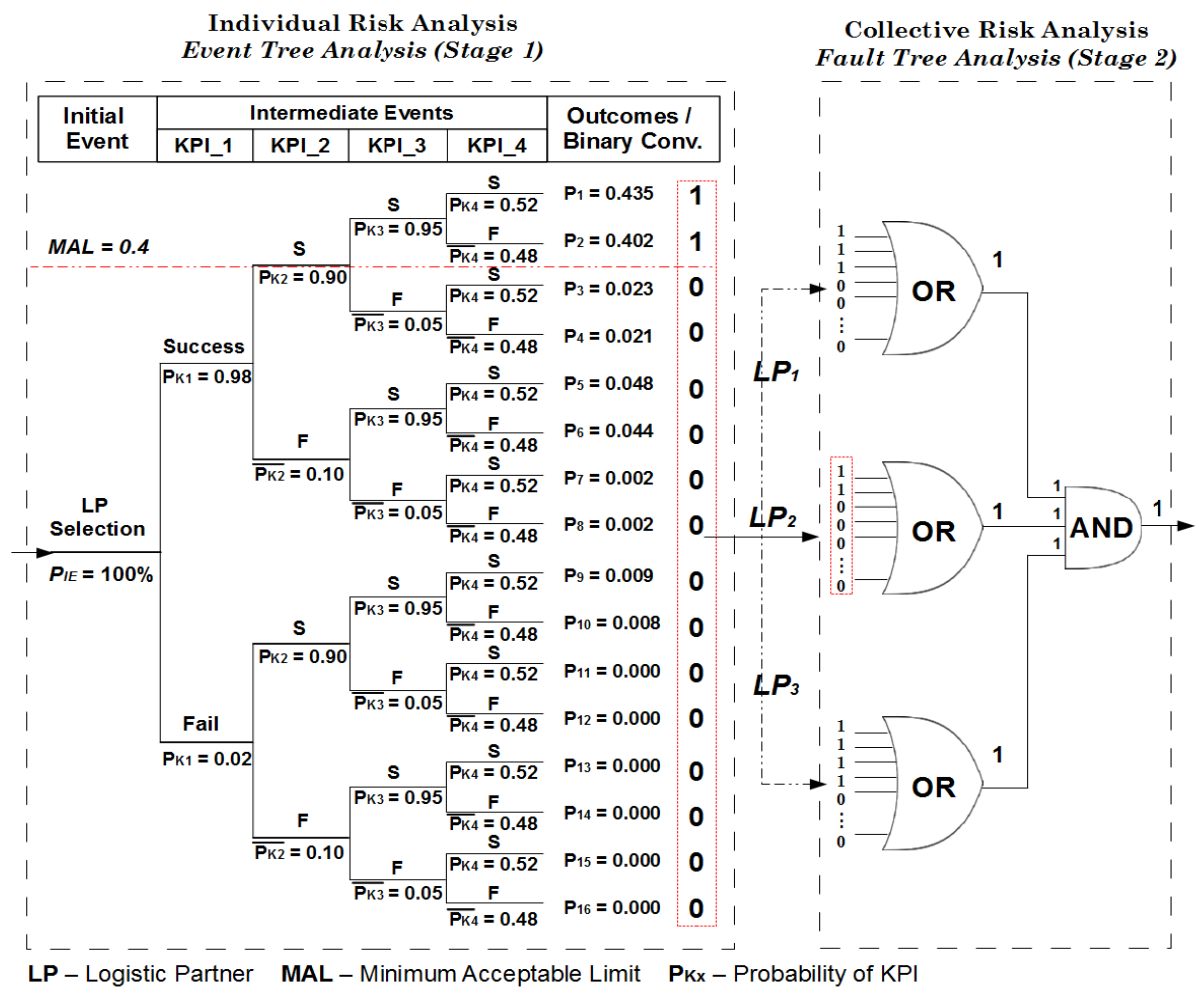

Fig. 3. MRALP method general example 
The values assigned to each KPI can vary from 0 to 1 and are associated with a success rate between 0 and 1 . Figure 4 shows a graph with hypothetic KPI values about trust associated to a LP, assuming it has participated in $n_{p a}$ past VOs. Since $n_{>m p s}$ represents the number of its previous participation in VOs and considering its KPIs values greater than MPS (with an '*'), Equation 1 presents the KPI success rate.

$$
\boldsymbol{P r}(\boldsymbol{K})=\frac{\boldsymbol{n}_{>m p s}}{\boldsymbol{n}_{\boldsymbol{p} \boldsymbol{a}}}
$$

The failure rate for a given KPI is represented as $\operatorname{Pr}(\bar{K})$ by the following equation:

$$
\operatorname{Pr}(\bar{K})=1-\operatorname{Pr}(K)
$$

That success and failure calculation procedure is repeated for all KPIs that compose the ET for a LP. A set of probabilities representing the success and failure probability for each KPI is obtained as a result, which are presented in Figure 3 by the four intermediate (and independent from each other) events KPI_1:4 that populate the ET. Event KPI_2, for instance, would be related to KPI communication, with fail and success values of 0,4 and 0,6 , respectively.

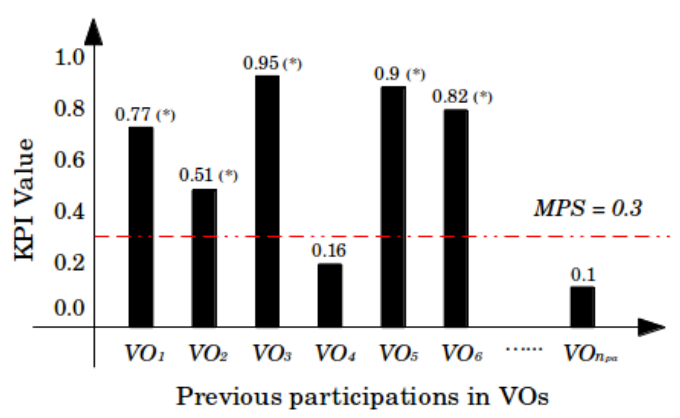

Fig. 4. Trust KPI historic values for a given LP

After assigning all probabilities for all ET branches, it is necessary to identify if the LPs are minimally qualified to compose a VO. For this, a calculation is performed to obtain the final probabilities for all event combinations composing the ET. They are determined for each of the $2^{|K|}$ branches of ET and are got by multiplying the probabilities of events that compose each path. The results greater than MAL are then selected to be part of the Stage 2 of MRALP.

The above concepts can be formalized as follows:

Let $L P=\left\{L P_{1}, L P_{2}, \cdots, L P_{p}\right\}$ be a set of $p$ LPs previously selected, where each element in this set is associated with a different type of logistic activity that is being requested in a business. Let $K=\left\{K_{1}, K_{2}, \cdots, K_{n}\right\}$ be a set of $n$ KPIs associated to a LP, and $\rho(K)$ the probability function associated with each event in $K$ (as defined in Equation 1). ETA events occur independently, i.e., where the occurrence of an event does not affect the occurrence of other event. This situation can be represented by the equalities defined in Equation (3) and Equation (4): 


$$
\begin{gathered}
\rho\left(K_{n}\left|K_{n-1}\right| \cdots \mid K_{1}\right)=\rho\left(K_{n}\right) \\
\rho\left(K_{1} \cap \cdots \cap K_{n}\right)=\rho\left(K_{1}\right) * \ldots * \rho\left(K_{n}\right)
\end{gathered}
$$

Now consider $P=\left\{P_{1}, P_{2}, \cdots, P_{2^{|K|} \mid}\right\}$ as a set of all possible outcomes from the $2^{|K|}$ ET events combinations. The procedure for obtaining this set was performed using a binary search tree [7], which travels $2^{|K|}$ different paths and assign a value to each element of $P$, as shown in Equation (5):

$$
P=\bigcup_{k=1}^{2^{|K|}}\left[\text { Initial Event } * \prod_{l=1}^{|K|} \omega(i, j, k, l)\right]
$$

The function $\omega$, as shown in Equation (6), corresponds to a 4-dimensional vector which performs a binary search in the tree, returning a path element of each iteration. Values $i$ and $j$ correspond to the beginning and ending of the search, and have initial values of $i=0$ and $j=2^{|K|}$. The value $k$ corresponds to the index of the sought element (an element of $P$ ) and $l$, the current level of the tree. The sequence of events can be viewed in Figure 3 (Stage 1).

$$
\omega(i, j, k, l)=\left\{\begin{aligned}
\operatorname{Pr}\left(K_{l}\right) ; j=(i+j) / 2, & k \leq(i+j) / 2 \\
1-\operatorname{Pr}\left(K_{l}\right) ; i=(i+j) / 2, & k>(i+j) / 2
\end{aligned}\right.
$$

After defined all possible outputs $P$ for a LP and calculated their probabilities, the method applies a constraint variable $Q$, which checks if its value is greater than or equal to MAL for each element of $P$. Only the results that are greater than MAL are considered, and the other are discarded. Thus, $Q=\left\{q_{1}, q_{2}, \cdots, q_{m}\right\}$ is a subset of P:

$$
Q=\{q \in P \mid q \geq M A L\}
$$

The final probability values obtained through Equation (7) will be used to measure and analyze the LP's risk. Consider the risk level of a LP defined by a pair $\langle R, S\rangle$. $R$ represents a condition of the LP to compose a VO (using boolean logic), while $S$ represents the success probability of the LP associated with the condition R. So $L P_{i}=\left\langle R_{i}, S_{i}\right\rangle$ for the $\mathrm{i}$-th selected LP and the i-th is the score $S$ associated with the ith result $R . R$ is calculated checking if $Q$ set has some element (Equation (8)), i.e. if there is at least one outcome value greater than MAL. A value 1 corresponds to the presence of elements so enabling the $L P$ to compose a VO. $S$ (Equation (9)) is calculated summing all elements of $Q$, obtaining the success probability rate for a LP.

$$
R= \begin{cases}0, & |Q|=0 \\ 1, & |Q| \neq 0\end{cases}
$$

$$
S=\sum_{i=1}^{|Q|} q_{i}
$$




\subsection{Collective Risk Analysis}

This second phase of the MRALP method aggregates the results provided by the first phase (i.e. the risk level of each pre-selected LPs) to calculate the VO success probability as a whole, i.e. if the VO formation can succeed or not.

This phase applies FTA (fault tree analysis) method [7]. FTA uses a logical diagram- called Fault Tree (FT) - which is a graphical representation of failure events logic that can occur in a system among all other possible event combinations. The graphical model can be translated into boolean logic using logical gates to calculate failures. Events are associated with input lines from the logic ports (0-failure, 1success) and must be analyzed to determine the logical connection between underlying failure events that might cause them.

FTA method takes the boolean values from ETA (first phase) as input (see Figure 3). An $O R$ logic gate with $2^{|K|}$ entries is associated to each LP, meaning that the number of OR logic gates changes according to the number of LPs.

Resulting values from OR logic gates are aggregated to an AND logic gate, which verifies if all LPs are able to compose the VO. This gate returns 1 on success and 0 on failure, i.e., if at least one LP is not able to compose the VO, the VO formation process is considered unfeasible. On the contrary, if all members are considered able to, the $\mathrm{VO}$ as a whole is considered able to go for operation.

For this, it is necessary to perform a calculation with all elements of $L P$ and, after that, to set a $R_{f}$, which corresponds to the success/failure value of the association between the LPs (Equation 10).If $R_{f}=1$ then the VO formation is considered feasible from the risk analysis point of view. If not, that $\mathrm{VO}$ is discarded and the procedure is restarted with other LPs, i.e. with the other (possible) VO compositions.

$$
R_{f}=\bigwedge_{i=1}^{|S P|} S P\left\langle R_{i}\right\rangle
$$

\section{$4 \quad$ An Illustrative Example}

This section presents an illustrative example to better show the proposed method. Suppose that a CO was created and three LPs $\left(\left\{L P_{1}, L P_{2}, L P_{3}\right\}\right)$ were selected to link four industrial partners (using the method developed in [4]. The goal is to measure the risky of every selected possible LP coalition for the given VO. Following the proposed method, for each LP coalition the individual risk of every LP is firstly measured and the overall VO risk is calculated afterwards.

Considering size restrictions, the risk analysis that will be illustrated will only consider $L P_{1}$, with the final values of $L P_{2}$ and $L P_{3}$ arbitrarily defined.

The assessment criteria $K$ of each LP are defined by a set of four KPIs: Trust $\left(K_{1}\right)$, Communication $\left(K_{2}\right)$, Collaboration $\left(K_{3}\right)$ and Commitment $\left(K_{4}\right)$. Table 1 shows 
hypothetical historical values that would be assigned to $L P_{1}{ }^{\prime} \mathrm{s}$ KPIs related to its participation in the last seven VOs. Equation (1) calculates the success probability of these KPIs applying a MPS=0.6.

Table 1. Quantitative values of KPIs according historical values of $L P_{1}$ in VOs

(*) KPI values greater than MPS $=0.6$ are considered in the risk analysis.

\begin{tabular}{|c|c|c|c|c|c|c|c|}
\hline & $\mathrm{OV}_{1}$ & $\mathrm{OV}_{2}$ & $O V_{3}$ & $\mathrm{OV}_{4}$ & $O V_{5}$ & $O V_{6}$ & $O V_{7}$ \\
\hline$K_{1}$ & $0.76\left(^{*}\right)$ & $0.72\left(^{*}\right)$ & 0.51 & $0.92\left(^{*}\right)$ & $0.94\left(^{*}\right)$ & 0.52 & $0.80\left(^{*}\right)$ \\
\hline$K_{2}$ & $0.93\left(^{*}\right)$ & $0.63\left(^{*}\right)$ & $0.61\left(^{*}\right)$ & $0.75\left(^{*}\right)$ & 0.55 & $0.70\left(^{*}\right)$ & $0.61\left(^{*}\right)$ \\
\hline$K_{3}$ & 0.55 & $0.60\left(^{*}\right)$ & $0.93\left(^{*}\right)$ & $0.88\left(^{*}\right)$ & $0.80\left(^{*}\right)$ & $0.98\left(^{*}\right)$ & $0.66\left(^{*}\right)$ \\
\hline$K_{4}$ & 0.52 & 0.51 & $0.60\left({ }^{*}\right)$ & $0.91\left(^{*}\right)$ & $0.87\left({ }^{*}\right)$ & $0.84\left({ }^{*}\right)$ & 0.54 \\
\hline
\end{tabular}

In order to individually measure its risk level, $L P_{1}$ is submitted to the first stage of MRALP method, applying ETA method. It should also consider the success and failure probabilities of each KPI that composes the intermediate events and then adding them as parameters in the ET. The ET graphical representation can be viewed in Figure 3 (Stage 1). According to Table 1, using Equations (1) and (2) and considering, for example, MPS $=0.6$, the success and failure probabilities associated with each KPI are calculated (Table 2) and the respective ET is formed.

Table 2. Success and failure probabilities to LP1

\begin{tabular}{|l|c|}
\hline Success Probabilities & Failure Probabilities \\
$\operatorname{Pr}\left(K_{1}\right)=5 / 7=0.71$ & $\operatorname{Pr}\left(\bar{K}_{1}\right)=1-0.71=0.29$ \\
$\operatorname{Pr}\left(K_{2}\right)=6 / 7=0.86$ & $\operatorname{Pr}\left(\bar{K}_{2}\right)=1-0.86=0.14$ \\
$\operatorname{Pr}\left(K_{3}\right)=6 / 7=0.86$ & $\operatorname{Pr}\left(\bar{K}_{3}\right)=1-0.86=0.14$ \\
$\operatorname{Pr}\left(K_{4}\right)=4 / 7=0.57$ & $\operatorname{Pr}\left(\bar{K}_{4}\right)=1-0.57=0.43$ \\
\hline
\end{tabular}

Let $P=\left\{P_{1}, P_{2}, \cdots, P_{16}\right\}$ be a set of all combinations among $K_{1}, K_{2}, K_{3}, K_{4}$ and, for example, MAL $=0.1$. Table 3 presents this result after applying Equation 5 . It represents the $2^{|K|}$ combinations of $K$, corresponding to all the probabilities (sixteen) associated with each event.

Next step consists in assigning a $\mathrm{S}$ score and a $\mathrm{R}$ boolean result for the $L P_{1}$ (Equations (8) and (9)). A constraint $\mathrm{Q}=\{0.3,0.15,0.12\}$ is defined (Equation (7)), corresponding to the probabilities of values to be greater than MAL.

$$
\begin{gathered}
S=(0.3+0.15+0.12)=0.57 \\
|Q|>0 \rightarrow R=1
\end{gathered}
$$


Table 3. Results from the event combinations

$(*)$ values greater than $\mathrm{MAL}=0.1$

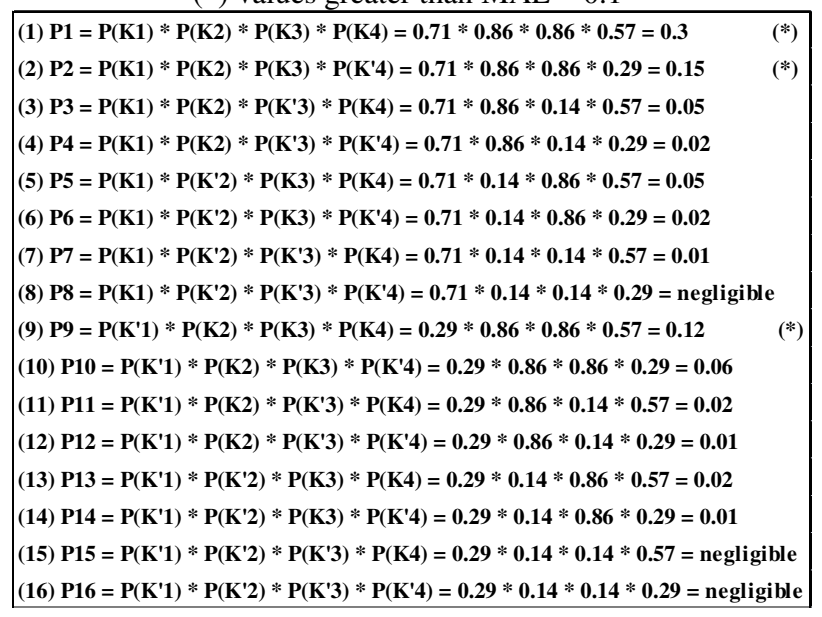

It can be seen that logistic partner $L P_{1}$ is able to compose a VO as it has at least one value greater than MAL. Now, (arbitrary) values of $\mathrm{R}$ and $\mathrm{S}$ are set to $L P_{2}$ and $L P_{3}$, as shown in Table 4.

Table 4. Values of $\langle R, S\rangle$ associated each logistic partner

\begin{tabular}{|c|c|c|c|}
\hline & $L P_{1}$ & $L P_{2}$ & $L P_{3}$ \\
\hline$\left\langle R_{i}, S_{i}\right\rangle$ & $\langle 1,0.47\rangle$ & $\langle 1,0.54\rangle$ & $\langle 1,0.38\rangle$ \\
\hline
\end{tabular}

The second stage of the method consists in aggregating all the individual results from the LP group and in analyzing them as a whole. This is done using FT results as input to verify if that VO coalition, collectively, is feasible or not. Equation (9) is applied considering the provided values (Table 4) using the $L P_{i}(R)$ :

$$
R_{f}=L P_{1}(R) \wedge L P_{2}(R) \wedge L P_{3}(R)=1 \wedge 1 \wedge 1=1
$$

As explained in Section 3, considering that $R_{f}=1$, this would mean that the combination of those three logistic partners has an acceptable level of risk. Therefore, they could become members of the VO and hence the VO could be created.

\section{Conclusions}

This paper has presented a supporting method to perform a risk analysis upon a set of logistic partners (LP) that are going to compose - together with industrial partners - a Virtual Organization (VO). It corresponds to an extension of a previous work, which selected LPs but without considering risks. This method represents the result of an ongoing research which aims at last to develop a comprehensive framework for VO risk analysis. 
The presented method, called MRALP, performs the risk analysis along two stages, firstly evaluating risks individually, per partner, and secondly at a global level. Four KPI are used as the essential basis for the selecting criteria, applying ETA (Event Tree Analysis) method in the first stage and FTA (Fault Tree Analysis) method in the second stage.

Although implemented in a controlled computing environment and with hypothetical values, the achieved results seemed promising about the suitability of the method regarding its purpose. Both ETA and FTA could model the required variables as long as it was needed besides being not much complex to use them.

Considering the state-of-the-art, the presented method proposed a concrete way to express to measure risks in a VO scenario when considering logistics. Yet, modeled as a process, it represents a systematic way to analyze risks. This is important once partners are autonomous and are members of long-term alliances so transparency in the involved processes is crucial for trust building. Moreover, being a more organized process, it has the potential to increase the agility in the VO formation process. On the other hand, it is assumed that a VO manager is the one responsible for the decision (i.e. the very final selection of LPs) as well as for actions aiming at mitigating risks, both in the creation and operation phases of the VO life cycle.

The four chosen KPIs (communication, commitment, trust and collaboration) to cope with risks in VO seem appropriate regarding literature. However, it is necessary to assess them in near-real scenarios, not only in terms if they are suitable enough or if more KPIs are required, but also to evaluate the complexity to implement them. Companies are often very much variable and this implementation also depends on the culture and working methods currently applied by the involved organizations.

Considering the combinatorial essence of the method, its computation can become overwhelming when dealing with very large global logistics chains, composed of tens of partners.

Although preliminary devoted to logistics and partners' selection problem, the method can be easily adapted to other application domains, basically changing the group of selected KPIs and the way they are calculated.

Next main steps include testing the method in near real scenarios as well as to extend it towards comprising the VO entirely, involving industrial partners. Besides that, a more detailed categorization of types of LPs (inbound and outbound as well as $4 \mathrm{PL}$ ) will help in a more precise definition of the most suitable LPs.

Acknowledgements. This work has been supported by the Brazilian and German research funding agencies Capes and DFG in the scope of the BRAGECRIM Program, project No. 2009/2. Also by the Brazilian research funding agency CNPq.

\section{References}

1. Mentzer, J.T., Konrad, B.P.: An efficiency/effectiveness approach to logistics performance analysis. Journal of Business Logistics 12, 33-62 (2006)

2. Mowshowitz, A.: Virtual Organization. ACM Communications 40, 30-37 (1997)

3. Camarinha-Matos, L.M., Afsarmanesh, H.: On reference models for collaborative networked organizations. Int. J. Production Research 46, 2453-2469 (2008) 
4. Alves Junior, O., Rabelo, R.J.: A KPI model for logistics partners's search and suggestion to create virtual organizations. International Journal of Networking and Virtual Organisations 12(2), 149-177 (2013)

5. Ryclik, I., Ryden, J.: Probability and risk analysis. Springer (2006)

6. Grabowski, M., Roberts, K.H.: Risk Mitigation in Virtual Organizations. Journal of Computer-Mediated Communication 10, 704-721 (1999)

7. Vose, D.: Risk Analysis: A quantitative analysis, 3rd edn. John Wiley \& Sons (2008)

8. Ericson, C.A.: Hazard Analysis Techniques for System Safety. John Wiley\&Sons (2005)

9. Bentley, J.L.: Multidimensional binary search trees used for associative searching. Communications of ACM 18, 509-517 (1975)

10. Rychlik, I., Rydén, J.: Probability and risk analysis: an introduction for engineers. Springer, Berlin (2006)

11. Rhee, S.J., Ishii, K.: Using cost based FMEA to enhance reliability and serviceability. Advanced Engineering Informatics 17(3), 179-188 (2003)

12. Bertsche, B.: Reliability in automotive and mechanical engineering. Springer (2008)

13. Alawamleh, M., Popplewell, K.: Risk Sources Identification in Virtual Organisation. Enterprise Interoperability, 265-277 (2010)

14. Alawamleh, M., Popplewell, K.: Analysing virtual organisation risk sources: An analytical network process. Int. J. Networking and Virtual Organisations 10, 18-39 (2012)

15. Li, Y., Liao, X.: Decision support for risk analysis on dynamic alliance. Journal Decision Support Systems 42, 2043-2059 (2007)

16. Baldo, F., Rabelo, R.J., Vallejos, R.V.: A framework for selecting performance indicators for virtual organisation partners' search and selection. International Journal of Production Research 27(17), 4737-4755 (2009)

17. Mosleh, A., Dias, A., Eghbali, G., Fazen, K.: An integrated framework for identification, classification and assessment of aviation system hazards. In: Proc. Int. Conf. on Probabilistic Safety Assessment and Management, Berlin, Germany, pp. 1-6 (1997)

18. Neely, A., Richards, H., Mills, J., Platts, K., Bourne, M.: Designing performance measures: a structured approach. Int. J. Op. \& Production Manag. 17, 1131-1152 (1997) 